\title{
Synchronization of Hindmarsh-Rose neurons: a numerical study
}

\author{
R. Erichsen Jr., M. S. Mainieri and L. G. Brunnet
}

Instituto de Física - Universidade Federal do Rio Grande do Sul, Porto Alegre, Brazil

The Hindmarsh-Rose model of neurons is a model that describes the essential of the spiking activity of biological neurons. In this work we present an exploratory numerical study of the time activities of two HR neurons interacting through electrical synapses. The knowledge of this simple system is a first step towards the understanding of the cooperative behavior of large neural assemblies. Several periodic and chaotic attractors were identified, as the coupling strength is increased from zero till the perfect synchronization regime. In addition to the known phase locking synchronization at weak coupling, electrical synapses also allow for both inphase and anti-phase synchronization from moderate to strong coupling. A regime where the system changes apparently randomly between in-phase and anti-phase locking evolves to a bistability regime, where both in-phase and anti-phase periodic attractors are locally stable. At the strong coupling regime in-phase chaotic evolution dominates, but windows with complex periodic behavior are also present.

\section{The effect of topology on neural networks with unstable memories}

\author{
S. Johnson, J. Marro and J. J. Torres \\ Institute "Carlos I" for Theoretical and Computational Physics, University of Granada, \\ Fuentenueva s/n, E-18071, Granada, Spain
}

We study an attractor neural network in which a noise parameter, meant to mimic synaptic depression, causes instabilities of the memory stored patterns leading to chaotic behaviour $[1,2]$. We studied the emergent properties of that network for different scale-free topologies of the network connectivity. As a consequence of the noise, three distinct phases emerge: a ferromagnetic or memory phase, a phase of chaotic hopping in which the activity of the network is continously jumping among the attractors, and a phase of periodic jumping between attractors. Using a standard mean-field analysis and for a single pattern, the dynamics of the network is reduced to an one-dimensional discrete map where the exponent of the power law connectivity distribution is a relevant parameter. Analysis of that map shows the existence of an optimal exponent, slightly larger than 2, which maximizes the range for the noise parameter in which the ferromagnetic type of behaviour (memory phase) is stable. On the contrary, for exponents smaller than 1.5, the larger range for the noise parameter occurs for the chaotic phase. We conclude that there are particular topologies which allows for good performance in memory 
Copyright of AIP Conference Proceedings is the property of American Institute of Physics and its content may not be copied or emailed to multiple sites or posted to a listserv without the copyright holder's express written permission. However, users may print, download, or email articles for individual use. 